\title{
Super connectivity of direct product of graphs*
}

\author{
Jin-Xin Zhou \\ Department of Mathematics, Beijing Jiaotong University, \\ Beijing 100044, P.R. China
}

Received 15 July 2012, accepted 14 June 2014, published online 23 January 2015

\begin{abstract}
For a graph $G, \kappa(G)$ denotes its connectivity. A graph $G$ is super connected, or simply super- $\kappa$, if every minimum separating set is the neighborhood of a vertex of $G$, that is, every minimum separating set isolates a vertex. The direct product $G_{1} \times G_{2}$ of two graphs $G_{1}$ and $G_{2}$ is a graph with vertex set $V\left(G_{1} \times G_{2}\right)=V\left(G_{1}\right) \times V\left(G_{2}\right)$ and edge set $E\left(G_{1} \times G_{2}\right)=$ $\left\{\left(u_{1}, v_{1}\right)\left(u_{2}, v_{2}\right) \mid u_{1} u_{2} \in E\left(G_{1}\right), v_{1} v_{2} \in E\left(G_{2}\right)\right\}$. Let $\Gamma=G \times K_{n}$, where $G$ is a non-trivial graph and $K_{n}(n \geq 3)$ is a complete graph on $n$ vertices. In this paper, we show that $\Gamma$ is not super- $\kappa$ if and only if either $\kappa(\Gamma)=n \kappa(G)$, or $\Gamma \cong K_{\ell, \ell} \times K_{3}(\ell>0)$.
\end{abstract}

Keywords: Super connectivity, direct product, vertex-cut.

Math. Subj. Class.: 05C40, $05 C 76$

\section{Introduction}

Throughout this paper only undirected simple connected graphs without loops and multiple edges are considered. Unless stated otherwise, we follow Bondy and Murty [4] for terminology and definitions.

Let $G=(V(G), E(G))$ be a graph. For two vertices $u, v \in V(G), u \sim v$ means that $u$ is adjacent to $v$ and $u v$ is the edge incident to $u$ and $v$ in $G$. The set of vertices adjacent to the vertex $v$ is called the neighborhood of $v$ and denoted by $N_{G}(v)$, i.e., $N_{G}(v)=\{u \mid u v \in E(G)\}$. The degree of $v$ is equal to $\left|N_{G}(v)\right|$, denoted by $d_{G}(v)$. The number $\delta(G)=\min \left\{d_{G}(v) \mid v \in V(G)\right\}$ is the minimum degree of $G$. For a subset $S \subseteq V(G)$, the subgraph induced by $S$ is denoted by $G[S]$. As usual, $K_{m, m}$, ( $m$ is a positive integer) denotes the complete bipartite graph; $K_{m, m}-m K_{2}$ denotes the graph obtained by removing a 1 -factor from $K_{m, m} ; K_{n}$ denotes the complete graph on $n$ vertices; and $\mathbb{Z}_{n}$ denotes the ring of integers modulo $n$.

* This work was supported by the National Natural Science Foundation of China (11271012).

E-mail address: jxzhou@bjtu.edu.cn (Jin-Xin Zhou) 
A separating set of a graph $G$ is a set of vertices whose deletion either disconnects $G$ or reduces $G$ to the trivial graph $K_{1}$. The connectivity of the graph $G$ is the minimum number of vertices in a separating set of $G$, and will be denoted by $\kappa(G)$. In particular, $\kappa\left(K_{n}\right)=$ $n-1$, and $\kappa(G)=0$ if and only if $G$ is disconnected or a $K_{1}$. Clearly, $\kappa(G) \leq \delta(G)$. A graph $G$ with minimum degree $\delta(G)$ is maximally connected if $\delta(G)=\kappa(G)$.

An interconnection network is often modeled as a graph $G$, where $V(G)$ is the set of processors and $E(G)$ is the set of communication links in the network. The connectivity $\kappa(G)$ of $G$ is an important measurement for fault-tolerance of the network, and the larger $\kappa(G)$ is, the more reliable the network is. As more refined indices of reliability than connectivity, super connectivity was proposed in [2,3]. A graph $G$ is super connected, super- $\kappa$, for short if every minimum separating set isolates a vertex of $G$.

The direct product $G_{1} \times G_{2}$ of two graphs $G_{1}$ and $G_{2}$ is defined as the graph with vertex set $V\left(G_{1}\right) \times V\left(G_{2}\right)$ and edge set $\left\{\left(u_{1}, v_{1}\right)\left(u_{2}, v_{2}\right) \mid u_{1} u_{2} \in E\left(G_{1}\right), v_{1} v_{2} \in E\left(G_{2}\right)\right\}$. The direct product is also called the Kronecker product, tensor product, cross product, categorical product, or conjunction. As an operation on binary relations, the direct product was introduced by Whitehead and Russell in their Principia Mathematica [21]. It is also equivalent to the direct product of the adjacency matrices of the graphs (see [20]). As one of the four standard graph products [11], the direct product has been studied from several points of view (see, for example, [1, 6, 8, 12, 13, 15]).

The connectivity of the direct product of graphs has also been investigated in several recent publications. For example, Brešar and Špacapan [7] obtained an upper bound and a lower bound on the edge-connectivity of the direct products with some exceptions, and they also obtained several upper bounds on the vertex-connectivity of the direct products of graphs. Mamut and Vumar [14] proved that $\kappa\left(K_{m} \times K_{n}\right)=(m-1)(n-1)$ where $m \geq n \geq 2$. In [9], it was shown that if $n \geq 3$ and $G$ is a bipartite graph, then $\kappa\left(G \times K_{n}\right)=$ $\min \{n \kappa(G),(n-1) \delta(G)\}$, and furthermore, the authors also conjectured that this is true for all nontrivial graph $G$. Later, this conjecture was confirmed independently by Wang and $\mathrm{Wu}[17]$ and Wang and Xue [18].

More recently, several papers dealing with the super-connectivity of direct product of graphs were published. Guo et al. [10] showed that for a bipartite graph $G$ with $\kappa(G)=$ $\delta(G), G \times K_{n}(n \geq 3)$ is super- $\kappa$. In [19], the authors generalized this result by showing that for a nonbipartite graph $G$ with $\kappa(G)=\delta(G), G \times K_{n}(n \geq 3)$ is super- $\kappa$. In [19], the authors also pointed out that Guo et al.'s result is not true when $G=K_{\ell, \ell}(\ell \geq 1)$ and $n=3$, and they also claimed that except for this case, Guo et al.'s statement is true.

The aim of this article is to determine all graphs $G$ such that $G \times K_{n}(n \geq 3)$ is not super- $\kappa$. The following is the main result.

Theorem 1.1. Let $\Gamma=G \times K_{n}$, where $n \geq 3$ and $G$ is a non-trivial graph. Then $\Gamma$ is not super- $\kappa$ if and only if one of the following happens.

(1) G has a minimum separating set $T$ so that $T \times V\left(K_{n}\right)$ is a minimum separating set of $\Gamma$. In particular, $\kappa(\Gamma)=n \kappa(G)$.

(2) $\Gamma \cong K_{\ell, \ell} \times K_{3}(\ell>0)$.

From Theorem 1.1 we can immediately obtain the following corollaries.

Corollary 1.2. [17, 18] Let $\Gamma=G \times K_{n}$, where $n \geq 3$ and $G$ is a non-trivial graph. Then $\kappa(\Gamma)=\min \{n \kappa(G),(n-1) \delta(G)\}$. 
Corollary 1.3. [10, 19] For a maximally connected graph $G, G \times K_{n}(n \geq 3)$ is not super- $\kappa$ if and only if $n=3$ and $G \cong K_{\ell, \ell}(\ell>0)$.

\section{Proof of Theorem 1.1}

We start by introducing some notations.

\section{Notations.}

- $\Gamma:=G \times K_{n}$, where $n \geq 3$ and $G$ is a non-trivial graph.

- $V(G):=\left\{u_{i} \mid i \in \mathbb{Z}_{m}\right\}$.

- $V\left(K_{n}\right):=\mathbb{Z}_{n}$.

- $V_{i}:=\left\{u_{i}\right\} \times V\left(K_{n}\right), i \in \mathbb{Z}_{m}$.

- $S$ : a minimum separating set of $\Gamma$.

- $\Gamma-S:=\bigcup_{i=0}^{s-1} \Gamma_{i}$, where each $\Gamma_{i}$ is a connected component of $\Gamma-S$.

- $W_{i}:=V\left(\Gamma_{i}\right)$.

In the following Lemmas 2.1-2.5, we assume that $\Gamma$ is not super connected, and $S$ is a minimum separating set of $\Gamma$ with each component $\Gamma_{i}$ of $\Gamma-S$ having at least two vertices.

By the definition, we can obtain the following easy facts.

Lemma 2.1. (1) $\delta(\Gamma)=(n-1) \delta(G)$.

(2) For any $i \in \mathbb{Z}_{m}, V_{i}$ is an independent subset of $V(\Gamma)$.

(3) If $u_{i_{0}}$ is adjacent to $v_{i_{1}}$ in $G$, then $\left(u_{i_{0}}, j\right) \sim\left(u_{i_{1}}, k\right)($ in $\Gamma)$ if and only if $j \neq k$. In particular, $\Gamma\left[V_{i_{0}} \cup V_{i_{1}}\right] \cong K_{n, n}-n K_{2}$.

(4) Let $T$ be a separating set of $G$. Then $T \times V\left(K_{n}\right)$ is also a separating set of $\Gamma$. In particular, $|S|=\kappa(\Gamma) \leq \min \{n \kappa(G),(n-1) \delta(G)\}$.

(5) $s \geq 2$ and $\left|W_{i}\right| \geq 2$ for each $i \in \mathbb{Z}_{s}$.

Lemma 2.2. For each $\left(u_{i}, j\right) \in S,\left(u_{i}, j\right)$ has at least one neighbor in $W_{i}$ for each $i \in \mathbb{Z}_{s}$.

Proof. Suppose to the contrary that $\left(u_{i}, j\right)$ has no neighbors in $W_{i}$ for some $i \in \mathbb{Z}_{s}$. Set $S^{\prime}=S-\left\{\left(u_{i}, j\right)\right\}$. Then $W_{i}$ must be a component of $\Gamma-S^{\prime}$. This implies that $S^{\prime}$ is also a separating set of $\Gamma$, contrary to the minimality of $S$.

Lemma 2.3. For two components $W_{k}, W_{\ell}$, if there exist $\left(u_{i}, i^{\prime}\right) \in W_{k}$ and $\left(u_{j}, j^{\prime}\right) \in W_{\ell}$ such that $u_{i} \sim u_{j}($ in $G)$, then $i^{\prime}=j^{\prime}$ and $W_{k} \cap V_{i}=\left\{\left(u_{i}, i^{\prime}\right)\right\}$ and $W_{\ell} \cap V_{j}=\left\{\left(u_{j}, j^{\prime}\right)\right\}$.

Proof. Since $u_{i} \sim u_{j}($ in $G)$, it follows from Lemma 2.1 (3) that $\Gamma\left[V_{i} \cup V_{j}\right] \cong K_{n, n}-n K_{2}$. As $\Gamma-S$ is disconnected, there are no edges between $W_{k}$ and $W_{\ell}$. Consequently, $i^{\prime}=j^{\prime}$ and $W_{k} \cap V_{i}=\left\{\left(u_{i}, i^{\prime}\right)\right\}$ and $W_{\ell} \cap V_{j}=\left\{\left(u_{j}, j^{\prime}\right)\right\}$.

Lemma 2.4. Assume that for each $V_{i}$ there exists at most one $W_{j}$ such that $V_{i} \cap W_{j} \neq \emptyset$. Then $S=T \times V\left(K_{n}\right)$, where $T$ is a minimum separating set of $G$. In particular, $\kappa(\Gamma)=$ $n \kappa(G)$. 
Proof. We shall first show the following two claims.

Claim 1 If there exists an $i \in \mathbb{Z}_{m}$ such that $V_{i} \cap S \neq \emptyset$ and $V_{i} \not \subseteq S$, then $\left|V_{i} \cap S\right|=n-1$. Furthermore, for each $W_{j}$, there is a $V_{\ell}$ such that $\left|W_{j} \cap V_{\ell}\right|=1$.

By the assumption, there is a unique $j \in \mathbb{Z}_{s}$ such that $V_{i} \cap W_{j} \neq \emptyset$. By Lemma 2.2, for each vertex, say $\left(u_{i}, i^{\prime}\right)$, in $V_{i} \cap S$, there is at least one neighbor, say $\left(u_{\ell}, \ell^{\prime}\right)$, in each $W_{t}$ with $t \neq j$. By Lemma 2.3, $\left|V_{i} \cap W_{j}\right|=\left|V_{\ell} \cap W_{t}\right|=1$. From our assumption we know that $\left|V_{i} \cap S\right|=\left|V_{\ell} \cap S\right|=n-1$.

Claim 2 For each $i \in \mathbb{Z}_{m}$, either $V_{i} \cap S=\emptyset$ or $V_{i} \subseteq S$.

Suppose on the contrary that there exists an $i \in \mathbb{Z}_{m}$ such that $V_{i} \cap S \neq \emptyset$ and $V_{i} \nsubseteq S$. For each $j \in \mathbb{Z}_{s}$, let $\Omega_{j}=\left\{\ell \in \mathbb{Z}_{m}|| V_{\ell} \cap W_{j} \mid=1\right\}$, and set $n_{j}=\left|\Omega_{j}\right|$. By Claim 1 , $n_{j}>0$. Without loss of generality, assume that $n_{0} \leq n_{1} \leq \ldots \leq n_{s-1}$.

Assume that $W_{0} \subseteq \bigcup_{\ell \in \Omega_{0}} V_{\ell}$. Then for each $\left(u_{i}, \ell\right) \in W_{0}$, we have $\left|V_{i} \cap W_{0}\right|=1$. Combining this with Lemma 2.3, we have for a fixed $\left(u_{i}, \ell\right) \in W_{0}$, if $u_{k} \sim u_{i}($ in $G)$, then $\left|V_{k} \cap S\right| \geq n-1$. As $n \geq 3$, one has

$$
|S| \geq\left|V_{i} \cap S\right|+\sum_{u_{j} \in N_{G}\left(u_{i}\right)}\left|V_{j} \cap S\right| \geq n-1+\delta(G)(n-1)>\kappa(\Gamma) .
$$

A contradiction occurs.

Now assume that $W_{0} \nsubseteq \bigcup_{\ell \in \Omega_{0}}$. Let $U=\bigcup_{V_{i} \subseteq S} V_{i}, Z_{0}=\bigcup_{\ell \in \Omega_{0}} V_{\ell}$, and $Z_{1}=$ $\bigcup_{\ell \in \Omega_{1}} V_{\ell}$. Set $T=U \cup Z_{0}$. Clearly, $\left|Z_{0} \cap W_{0}\right|=n_{0}$. Since $n_{1} \geq n_{0}$ and $n \geq 3$, one has $\left|Z_{0} \cap W_{0}\right|=n_{0}<n_{1}(n-1)=\left|Z_{1} \cap S\right|$. Then

$$
\begin{aligned}
|T|=|U|+\left|Z_{0}\right| & =|U|+\left|Z_{0} \cap S\right|+\left|Z_{0} \cap W_{0}\right| \\
& <|U|+\left|Z_{0} \cap S\right|+\left|Z_{1} \cap S\right| \\
& \leq|S| .
\end{aligned}
$$

Since $S$ is a minimum separating set, $\Gamma-T$ is connected. So there is an edge between $W_{0} \backslash T$ and $V(\Gamma) \backslash\left(T \cup W_{0}\right)$. We may assume that $\left(v_{i}, j\right) \in W_{0} \backslash T$ is adjacent to $\left(v_{s}, k\right) \in V(\Gamma) \backslash\left(T \cup W_{0}\right)$. Obviously, $\left(v_{s}, k\right) \in S \backslash T$. Since $U=\bigcup_{V_{i} \subseteq S} V_{i}$ and $T=U \cup Z_{0}$, one has $V_{s} \nsubseteq S$. If $V_{s} \cap W_{0} \neq \emptyset$, then by Claim 1, we must have $\left|V_{s} \cap W_{0}\right|=1$ and so $V_{s} \subseteq Z_{0} \subseteq T$. This contradicts the fact that $\left(v_{s}, k\right) \in S \backslash T$. Consequently, $V_{s} \cap W_{0}=\emptyset$. It follows that $V_{s} \cap W_{t} \neq \emptyset$ for some $t>0$. Since $\left(v_{i}, j\right) \sim\left(v_{s}, k\right)$, by Lemma 2.3, $\left|V_{i} \cap W_{0}\right|=1$ and so $V_{i} \subseteq Z_{0} \subseteq T$. This contradicts the fact that $\left(v_{i}, j\right) \in W_{0} \backslash T$.

Now we are ready to finish the proof. From Claim 2 it follows that $S=T \times V\left(K_{n}\right)$ for some subset $T$ of $V(G)$. Since $n \geq 3, T$ is a separating set of $G$ (see [20]). So, $|S| \geq \kappa(G) n$. However, by Lemma $2.1(4),|S| \leq n \kappa(G)$. Hence, $|S|=n \kappa(G)$.

Lemma 2.5. Assume that there exist a $V_{i}$ and two different $W_{j_{0}}, W_{j_{1}}$ such that $V_{i} \cap W_{k} \neq \emptyset$ with $k=j_{0}, j_{1}$. Then $n=3$ and $G \cong K_{\ell, \ell}(\ell>0)$.

Proof. Recall that $W_{k}=V\left(\Gamma_{k}\right)$ with $k=j_{0}$ or $j_{1}$. We shall finish the proof by the following claims.

Claim $1 V(\Gamma)=W_{j_{0}} \cup W_{j_{1}} \cup S,\left|V_{i} \cap W_{j_{0}}\right|=\left|V_{i} \cap W_{j_{1}}\right|=1$ and $\left|V_{i} \cap S\right|=n-2$. 
By Lemma 2.1 (2), $V_{i}$ is an independent subset, and by Lemma 2.1 (5), $\left|W_{k}\right| \geq 2$ with $k=j_{0}$ or $j_{1}$. It follows that $V_{i} \cap W_{k} \subset W_{k}$. Since $\Gamma_{j_{0}}$ is connected, there exist $\left(u_{i}, t_{0}\right) \in V_{i} \cap W_{j_{0}}$ and $\left(u_{i_{0}}, t_{0}^{\prime}\right) \in W_{j_{0}} \backslash\left(V_{i} \cap W_{j_{0}}\right)$ such that $\left(u_{i}, t_{0}\right) \sim\left(u_{i_{0}}, t_{0}^{\prime}\right)$. Similarly, there exist $\left(u_{i}, t_{1}\right) \in V_{i} \cap W_{j_{1}}$ and $\left(u_{i_{1}}, t_{1}^{\prime}\right) \in W_{j_{1}} \backslash\left(V_{i} \cap W_{j_{1}}\right)$ such that $\left(u_{i}, t_{1}\right) \sim\left(u_{i_{1}}, t_{1}^{\prime}\right)$. From Lemma 2.3 we obtain that $t_{0}^{\prime}=t_{1}, V_{i} \cap W_{j_{0}}=\left\{\left(u_{i}, t_{0}\right)\right\}$ and $V_{i_{0}} \cap W_{j_{0}}=\left\{\left(u_{i_{0}}, t_{1}\right)\right\}$, and $t_{1}^{\prime}=t_{0}, V_{i} \cap W_{j_{1}}=\left\{\left(u_{i}, t_{1}\right)\right\}$ and $V_{i_{1}} \cap W_{j_{1}}=\left\{\left(u_{i_{1}}, t_{0}\right)\right\}$ (see Figure 1). In particular, we have $\left|V_{i} \cap W_{j_{0}}\right|=\left|V_{i} \cap W_{j_{1}}\right|=1$.

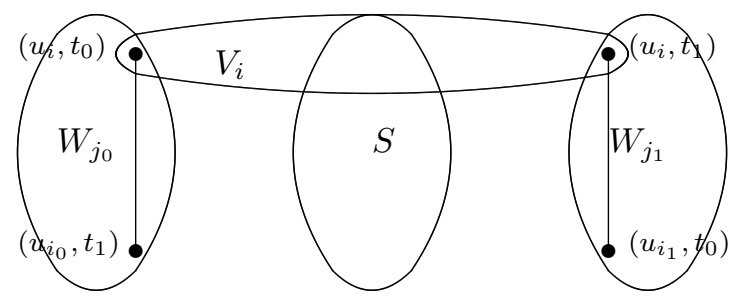

Figure 1: Explanation of the proof of Claim 1

It follows that $\left|V_{i} \cap S\right| \leq n-2$. If $\left|V_{i} \cap S\right|<n-2$, then we would have $V_{i} \cap W_{j} \neq \emptyset$ for some $j \neq j_{0}, j_{1}$. Take $\left(u_{i}, t\right) \in V_{i} \cap W_{j}$. Clearly, $t \neq t_{0}, t_{1}$. This forces that $\left(u_{i}, t\right) \sim\left(u_{i_{0}}, t_{1}\right)$, contrary to the fact that $\Gamma_{j_{0}}$ and $\Gamma_{j}$ are two distinct components. Thus, $\left|V_{i} \cap S\right|=n-2$.

At last, we shall show that $s=2$. Suppose to the contrary that $s>2$. Since $\left|V_{i} \cap S\right|=$ $n-2>0$, we can take $\left(u_{i}, j\right) \in V_{i} \cap S$. By Lemma 2.2, $\left(u_{i}, j\right)$ has a neighbor, say $\left(u_{k}, j^{\prime}\right)$ in each $W_{j}$ with $j \neq j_{0}, j_{1}$. Since $t_{0} \neq t_{1}$, either $\left(u_{k}, j^{\prime}\right) \sim\left(u_{i}, t_{0}\right)$ or $\left(u_{k}, j^{\prime}\right) \sim\left(u_{i}, t_{1}\right)$. This is again contrary to the fact that $\Gamma_{j_{0}}, \Gamma_{j_{1}}$ and $\Gamma_{j}$ are three distinct components. Thus, $s=2$ and hence $V(\Gamma)=W_{j_{0}} \cup W_{j_{1}} \cup S$.

By Claim 1, we may assume that $V_{i} \cap W_{j_{0}}=\left\{\left(u_{i}, t_{0}\right)\right\}$ and $V_{i} \cap W_{j_{1}}=\left\{\left(u_{i}, t_{1}\right)\right\}$.

Claim 2 For each $\left(u_{j}, \ell\right) \in N_{\Gamma}\left(\left(u_{i}, t_{0}\right)\right) \cap W_{j_{0}},\left|V_{j} \cap S\right|=n-1$ or $n-2$. There is at least one $\left(u_{i_{0}}, \ell_{0}\right) \in N_{\Gamma}\left(\left(u_{i}, t_{0}\right)\right) \cap W_{j_{0}}$ such that $\left|V_{i_{0}} \cap S\right|=n-2$.

Take $\left(u_{j}, \ell\right) \in N_{\Gamma}\left(\left(u_{i}, t_{0}\right)\right) \cap W_{j_{0}}$. From Claim 1 we see that $V_{i} \cap W_{j_{1}}=\left\{\left(u_{i}, t_{1}\right)\right\}$. By Lemma 2.3, we have $\left|V_{j} \cap W_{j_{0}}\right|=1$, implying $\left|V_{j} \cap S\right| \leq n-1$. If $\left|V_{j} \cap S\right|<n-1$ then we must have $V_{j} \cap W_{j_{1}} \neq \emptyset$. By Claim 1, we have $\left|V_{j} \cap S\right|=n-2$. Therefore, $\left|V_{j} \cap S\right|=n-1$ or $n-2$.

Suppose that for each $\left(u_{j}, \ell\right) \in N_{\Gamma}\left(\left(u_{i}, t_{0}\right)\right) \cap W_{j_{0}}$, we have $\left|V_{j} \cap S\right|=n-1$. Noting that $n \geq 3$, one has

$$
|S| \geq\left|V_{i} \cap S\right|+\sum_{u_{j} \in N_{G}\left(u_{i}\right)}\left|U_{j} \cap S\right| \geq n-2+\delta(G)(n-1)>\kappa(\Gamma),
$$

a contradiction. Thus, there is at least one $\left(u_{i_{0}}, \ell_{0}\right) \in N_{\Gamma}\left(\left(u_{i}, t_{0}\right)\right) \cap W_{j_{0}}$ such that $\mid V_{i_{0}} \cap$ $S \mid=n-2$.

Now we know that Claim 2 holds. Since $\left(u_{i_{0}}, \ell_{0}\right) \in N_{\Gamma}\left(\left(u_{i}, t_{0}\right)\right) \cap W_{j_{0}}$, it follows from Lemma 2.3 that $V_{i_{0}} \cap W_{j_{0}}=\left\{\left(u_{i_{0}}, t_{1}\right)\right\}$ and $V_{i_{0}} \cap W_{j_{1}}=\left\{\left(u_{i_{0}}, t_{0}\right)\right\}$ (see Figure 2). By the arbitrariness of $V_{i}$, Claims 1,2 also hold if we replace $V_{i}$ by $V_{i_{0}}$. 


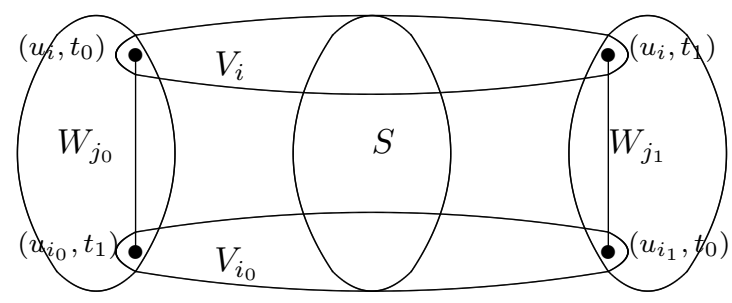

Figure 2: Explanation of Claims 1,2

For the convenience of statement, we shall use the following notations in the remainder of the proof.

\section{Notations}

(1) $N_{i}=N_{\Gamma}\left(\left(u_{i}, t_{0}\right)\right) \cap W_{j_{0}}$,

(2) $N_{i_{0}}=N_{\Gamma}\left(\left(u_{i_{0}}, t_{1}\right)\right) \cap W_{j_{0}}$,

(3) $\Omega_{i}=\left\{k \in \mathbb{Z}_{m} \mid V_{k} \cap N_{i} \neq \emptyset\right\}$,

(4) $\Omega_{i_{0}}=\left\{k \in \mathbb{Z}_{m} \mid V_{k} \cap N_{i_{0}} \neq \emptyset\right\}$,

(5) $\Delta_{i}=\left\{k \in \mathbb{Z}_{m} \mid k \notin \Omega_{i}, V_{k} \cap N_{\Gamma}\left(\left(u_{i}, t_{0}\right)\right) \neq \emptyset\right\}$,

(6) $\Delta_{i_{0}}=\left\{k \in \mathbb{Z}_{m} \mid k \notin \Omega_{i_{0}}, V_{k} \cap N_{\Gamma}\left(\left(u_{i_{0}}, t_{1}\right)\right) \neq \emptyset\right\}$.

It is easy to see that $N_{G}\left(u_{i}\right)=\left\{u_{k} \mid k \in \Omega_{i} \cup \Delta_{i}\right\}$ and $N_{G}\left(u_{i_{0}}\right)=\left\{u_{k} \mid k \in\right.$ $\left.\Omega_{i_{0}} \cup \Delta_{i_{0}}\right\}$. Hence, $\left|\Omega_{i}\right|+\left|\Delta_{i}\right|=d_{G}\left(u_{i}\right)$ and $\left|\Omega_{i_{0}}\right|+\left|\Delta_{i_{0}}\right|=d_{G}\left(u_{i_{0}}\right)$.

Claim $3\left|N_{i}\right|=\left|\Omega_{i}\right|$ and $\left|N_{i_{0}}\right|=\left|\Omega_{i_{0}}\right|$.

By Claim 1, for each $k \in \Omega_{i}$, we have $\left|V_{k} \cap W_{j_{0}}\right|=1$. It follows that $\left|N_{i}\right|=\left|\Omega_{i}\right|$. Similarly, $\left|N_{i_{0}}\right|=\left|\Omega_{i_{0}}\right|$.

Claim 4 Both $N_{i}$ and $N_{i_{0}}$ are independent subsets of $V(\Gamma)$.

Take any two vertices, say $\left(u_{i_{1}}, t\right),\left(u_{i_{2}}, t^{\prime}\right)$ in $N_{i}$. Since $V_{i} \cap W_{j_{1}}=\left\{\left(u_{i}, t_{1}\right)\right\}$, from Lemma 2.3 it follows that $t=t^{\prime}=t_{1}$. So, $\left(u_{i_{1}}, t\right)$ is not adjacent to $\left(u_{i_{2}}, t^{\prime}\right)$. Therefore, $N_{i}$ is an independent subset of $V(\Gamma)$. Similarly, $N_{i_{0}}$ is also an independent subset.

Claim $5\left(\bigcup_{k \in \Omega_{i} \cup \Delta_{i}} V_{k}\right) \cap\left(\bigcup_{k \in \Omega_{i_{0}}} V_{k}\right)=\emptyset$ and $\left(\bigcup_{k \in \Omega_{i_{0}} \cup \Delta_{i_{0}}} V_{k}\right) \cap\left(\bigcup_{k \in \Omega_{i}} V_{k}\right)=\emptyset$.

Suppose that $\left(\bigcup_{k \in \Omega_{i} \cup \Delta_{i}} V_{k}\right) \cap\left(\bigcup_{k \in \Omega_{i_{0}}} V_{k}\right) \neq \emptyset$. Take $\left(u_{j}, t\right) \in\left(\bigcup_{k \in \Omega_{i} \cup \Delta_{i}} V_{k}\right) \cap$ $\left(\bigcup_{k \in \Omega_{i_{0}}} V_{k}\right)$. Then $V_{j} \cap N_{i_{0}} \neq \emptyset$, implying that $V_{j} \cap W_{j_{0}} \neq \emptyset$. Assume $\left(u_{j}, t^{\prime}\right) \in V_{j} \cap W_{j_{0}}$. Clearly, $u_{i}$ and $u_{i_{0}}$ are neighbors of $u_{j}$ in $G$. Since $t_{0} \neq t_{1}$, either $\left(u_{j}, t^{\prime}\right) \sim\left(u_{i}, t_{1}\right)$ or $\left(u_{j}, t^{\prime}\right) \sim\left(u_{i_{0}}, t_{0}\right)$. This is contrary to the fact that there are no edges between $W_{j_{0}}$ and $W_{j_{1}}$. Thus, $\left(\bigcup_{k \in \Omega_{i} \cup \Delta_{i}} V_{k}\right) \cap\left(\bigcup_{k \in \Omega_{i_{0}}} V_{k}\right)=\emptyset$. Similarly, we have $\left(\bigcup_{k \in \Omega_{i_{0}} \cup \Delta_{i_{0}}} V_{k}\right) \cap$ $\left(\bigcup_{k \in \Omega_{i}} V_{k}\right)=\emptyset$.

Claim 6 Let $k \in \Delta_{i} \cup \Delta_{i_{0}}$. Then $V_{k} \cap W_{j_{0}}=\emptyset$ and $\left|V_{k} \cap S\right| \geq n-1$.

Assume $k \in \Delta_{i}$. Then $u_{k} \in N_{G}\left(u_{i}\right)$. If $V_{k} \cap W_{j_{0}} \neq \emptyset$, then take $\left(u_{k}, t\right) \in V_{k} \cap W_{j_{0}}$. Since $k \notin \Omega_{i},\left(u_{k}, t\right)$ is not adjacent to $\left(u_{i}, t_{0}\right)$, and hence $t=t_{0}$. Consequently, $\left(u_{k}, t\right) \sim$ $\left(u_{i}, t_{1}\right)$, a contradiction. Thus, $V_{k} \cap W_{j_{0}}=\emptyset$. By Lemma 2.3, $\left|V_{k} \cap W_{j_{1}}\right| \leq 1$, and hence $\left|V_{k} \cap S\right| \geq n-1$. With a similar argument, we can show that if $k \in \Delta_{i_{0}}$, then $V_{k} \cap W_{j_{0}}=\emptyset$ and $\left|V_{k} \cap S\right| \geq n-1$. 
Claim 7 (1) $n=3$; (2) $\left|N_{i}\right|=\left|N_{i_{0}}\right|=\delta(G)$; (3) $\left|\Delta_{i}\right|=\left|\Delta_{i_{0}}\right|=0$; (4) $|S|=2 \delta(G)$; (5) $S=\bigcup_{k \in \Omega_{i} \cup \Omega_{i_{0}}}\left(V_{k} \cap S\right)$; (6) for each $k \in \Omega_{i} \cup \Omega_{i_{0}},\left|V_{k} \cap S\right|=\left|V_{k} \cap W_{j_{0}}\right|=\left|V_{k} \cap W_{j_{1}}\right|=1$.

By the arbitrariness of $V_{i}$ and $V_{i_{0}}$, we may assume that $\left|N_{i}\right| \leq\left|N_{i_{0}}\right|$. By Claim 5 ,

$$
|S| \geq\left|\bigcup_{k \in \Omega_{i_{0}}}\left(V_{k} \cap S\right)\right|+\left|\left(\bigcup_{k \in \Omega_{i} \cup \Delta_{i}}\left(V_{k} \cap S\right)\right)\right| .
$$

By Claim 2, if $k \in \Omega_{i} \cup \Omega_{i_{0}}$, then $\left|V_{k} \cap S\right| \geq n-2$, and by Claim 6, if $k \in \Delta_{i} \cup \Delta_{i_{0}}$, then $\left|V_{k} \cap S\right| \geq n-1$. It follows that

$$
|S| \geq(n-2)\left|\Omega_{i_{0}}\right|+(n-2)\left|\Omega_{i}\right|+(n-1)\left|\Delta_{i}\right| .
$$

By Claim 3, we have

$$
|S| \geq(n-2)\left|N_{i_{0}}\right|+(n-2)\left|N_{i}\right|+(n-1)\left|\Delta_{i}\right| .
$$

Since $\left|N_{i_{0}}\right| \geq\left|N_{i}\right|$ and $n \geq 3$, we obtain that

$$
|S| \geq 2(n-2)\left|N_{i}\right|+(n-1)\left|\Delta_{i}\right| \geq(n-1) d_{G}\left(u_{i}\right) .
$$

However, by Lemma 2.1 (4), we have $|S| \leq(n-1) \delta(G)$. So, in the above four inequalities, "=" must hold. By Eq. (2.4) we obtain that $n=3,\left|N_{i}\right|=\left|N_{i_{0}}\right|$, and $\delta(G)=d_{G}\left(u_{i}\right)$. Furthermore, for each $k \in \Omega_{i} \cup \Omega_{i_{0}},\left|V_{k} \cap S\right|=n-1$, and for each $k \in \Delta_{i},\left|V_{k} \cap S\right|=n-1$. It follows that

$$
|S|=2\left|N_{i}\right|+2\left|\Delta_{i}\right|=2 \delta(G) .
$$

To show that $\left|\Delta_{i}\right|=\left|\Delta_{i_{0}}\right|=0$, we shall first show that

$$
\left(\bigcup_{k \in \Delta_{i}} V_{k}\right) \cap\left(\bigcup_{k \in \Omega_{i_{0}} \cup \Delta_{i_{0}}} V_{k}\right)=\emptyset .
$$

Suppose on the contrary that for some $k \in \Delta_{i}, V_{k} \cap\left(\bigcup_{k \in \Omega_{i_{0}} \cup \Delta_{i_{0}}} V_{k}\right) \neq \emptyset$. Since $\left|V_{k} \cap S\right|=$ $n-1$, from Claim 6 it follows that $\left|V_{k} \cap W_{j_{1}}\right|=1$. Take $\left(u_{k}, t\right) \in V_{k} \cap W_{j_{1}}$. Then $u_{i}, u_{i_{0}}$ are neighbors of $u_{k}$ in $G$. Since $t_{0} \neq t_{1}$, either $\left(u_{k}, t\right) \sim\left(u_{i}, t_{1}\right)$ or $\left(u_{k}, t\right) \sim$ $\left(u_{i_{0}}, t_{0}\right)$. This is contrary to the fact that there are no edges between $W_{0}$ and $W_{1}$. Thus, $\left(\bigcup_{k \in \Delta_{i}} V_{k}\right) \cap\left(\bigcup_{k \in \Omega_{i_{0}} \cup \Delta_{i_{0}}} V_{k}\right)=\emptyset$. It follows that

$$
\begin{aligned}
|S| & \geq\left|\bigcup_{k \in \Omega_{i_{0}} \cup \Delta_{i_{0}}}\left(V_{k} \cap S\right)\right|+\left|\left(\bigcup_{k \in \Omega_{i} \cup \Delta_{i}}\left(V_{k} \cap S\right)\right)\right| \\
& =\left|\bigcup_{k \in \Omega_{i_{0}}}\left(V_{k} \cap S\right)\right|+\left|\left(\bigcup_{k \in \Omega_{i} \cup \Delta_{i}}\left(V_{k} \cap S\right)\right)\right|+\left|\bigcup_{k \in \Delta_{i_{0}}}\left(V_{k} \cap S\right)\right| \\
& \geq 2\left|N_{i}\right|+2\left|\Delta_{i}\right|+2\left|\Delta_{i_{0}}\right| \\
& =2 \delta(G)+2\left|\Delta_{i_{0}}\right| .
\end{aligned}
$$

Combining this with Eq. (2.5) we obtain that $\left|\Delta_{i_{0}}\right|=0$. Since $d_{G}\left(u_{i}\right)=\delta(G)$, we have $d_{G}\left(u_{i_{0}}\right) \geq d_{G}\left(u_{i}\right)$. Recall that $\left|N_{i}\right|+\left|\Delta_{i}\right|=d_{G}\left(u_{i}\right)$ and $\left|N_{i_{0}}\right|+\left|\Delta_{i_{0}}\right|=d_{G}\left(u_{i_{0}}\right)$. Since $\left|N_{i}\right|=\left|N_{i_{0}}\right|$, one has $\left|\Delta_{i_{0}}\right| \geq\left|\Delta_{i}\right|$, implying $\left|\Delta_{i}\right|=0$.

At last, from Eq. (2.1) it can be deduced that

$$
S=\left(\bigcup_{k \in \Omega_{i_{0}}}\left(V_{k} \cap S\right)\right) \cup\left(\bigcup_{k \in \Omega_{i}}\left(V_{k} \cap S\right)\right) .
$$


Claim $8 W_{j_{0}}=N_{i} \cup N_{i_{0}}$.

Suppose that $W_{j_{0}} \neq N_{i} \cup N_{i_{0}}$. Since $\Gamma_{0}$ is a component of $\Gamma-S$, we can take a vertex, say $\left(v_{k_{1}}, t\right)$, in $W_{j_{1}}-\left(N_{i} \cup N_{i_{0}}\right)$ such that $\left(v_{k_{1}}, t\right)$ is adjacent to some vertex, say $\left(v_{k_{2}}, t^{\prime}\right)$, in $N_{i} \cup N_{i_{0}}$. Since $\left(v_{k_{2}}, t^{\prime}\right) \in N_{i} \cup N_{i_{0}}$, by Claim $7\left|V_{k_{2}} \cap W_{j_{1}}\right|=1$. By Lemma 2.3, we have $\left|V_{k_{1}} \cap W_{j_{0}}\right|=1$. By Claim 1, we have $V_{k_{1}} \cap S \neq \emptyset$. From Eq. (2.6) we see that $k_{1} \in \Omega_{i_{0}} \cup \Omega_{i}$, and hence $\left(v_{k_{1}}, t\right) \in N_{i} \cup N_{i_{0}}$, a contradiction. Thus, $W_{j_{0}}=N_{i} \cup N_{i_{0}}$.

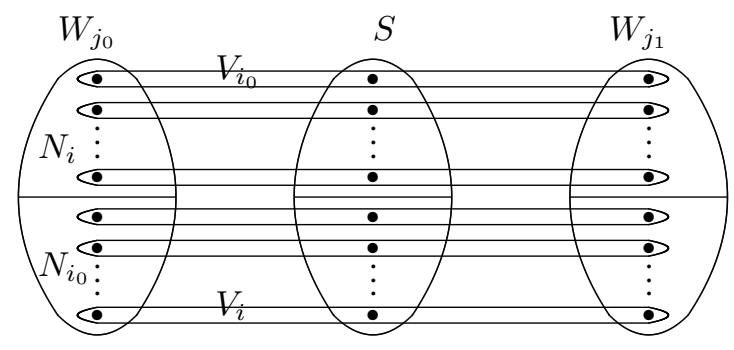

Figure 3: Explanation of Claims 8,9

Claim $9 m=|G|=2 \delta(G)$.

By Claim 8, $\left|W_{j_{0}}\right|=2 \delta(G)$. So, $m \geq\left|W_{j_{0}}\right|=2 \delta(G)$. Suppose that $m>2 \delta(G)$. By Claim 7, for each $k \in \Omega_{i} \cup \Omega_{i_{0}},\left|V_{k} \cap S\right|=\left|V_{k} \cap W_{j_{0}}\right|=\left|V_{k} \cap W_{j_{1}}\right|=1$, and $S=\bigcup_{k \in \Omega_{i_{0}} \cup \Omega_{i}}\left(V_{k} \cap S\right)$. This implies that $\bigcup_{k \in \Omega_{i_{0}} \cup \Omega_{i}}\left(V_{k} \cap W_{j_{1}}\right)$ is a proper subset of $W_{j_{1}}$. By the connectedness of $\Gamma_{1}$, take an edge $e$ in $\Gamma_{1}$ such that one end, say $\left(u_{k_{1}}, t\right)$, of $e$ is in $\bigcup_{k \in \Omega_{i_{0}} \cup \Omega_{i}}\left(V_{k} \cap W_{j_{1}}\right)$ and the other end, say $\left(u_{k_{2}}, t^{\prime}\right)$, is in $W_{j_{1}} \backslash \bigcup_{k \in \Omega_{i_{0}} \cup \Omega_{i}}\left(V_{k} \cap W_{j_{1}}\right)$. By Claim 7, $\left|V_{k_{1}} \cap W_{j_{0}}\right|=1$, and by Lemma 2.3, we have $\left|V_{k_{2}} \cap W_{j_{1}}\right|=1$. By Claim 1, $\left|V_{k_{2}} \cap S\right| \geq 1$. It follows from Eq. (2.6) that $k_{2} \in \Omega_{i_{0}} \cup \Omega_{i}$. This forces that $\left(u_{k_{2}}, t^{\prime}\right) \in$ $\bigcup_{k \in \Omega_{i_{0}} \cup \Omega_{i}}\left(V_{k} \cap W_{j_{1}}\right)$, a contradiction.

Claim $10 G \cong K_{\ell, \ell}$, where $\ell=\delta(G)$.

Clearly, $\left\{u_{k} \mid k \in \Omega_{i} \cup \Omega_{i_{0}}\right\} \subseteq V(G)$. By Claim 9, $m=|G|=2 \delta(G)$. It follows that $V(G)=\left\{u_{k} \mid k \in \Omega_{i} \cup \Omega_{i_{0}}\right\}$. Set $B_{0}=\left\{u_{k} \mid k \in \Omega_{i}\right\}$ and $B_{1}=\left\{u_{k} \mid k \in \Omega_{i_{0}}\right\}$. Take any two vertices, say $u_{k_{1}}$ and $u_{k_{2}}$, in $B_{0}$. Suppose $u_{k_{1}} \sim u_{k_{2}}$. By Claim 7, we may assume that $V_{k_{i}} \cap W_{j_{0}}=\left\{\left(u_{k_{i}}, d_{i}\right)\right\}$ with $i=1$ or 2 . From Claim 4 we obtain that $\left(u_{k_{1}}, d_{1}\right)$ is not adjacent to $\left(u_{k_{2}}, d_{2}\right)$, and hence $d_{1}=d_{2}$. Since $u_{k_{1}} \sim u_{k_{2}},\left(u_{k_{1}}, d_{1}\right)$ is adjacent to all the remaining vertices in $V_{k_{2}}$. Again, by Claim 7, we get that $\left|V_{k_{2}} \cap W_{j_{1}}\right|=1$. This implies that there is an edge between $W_{j_{0}}$ and $W_{j_{1}}$, a contradiction. Therefore, $u_{k_{1}}$ and $u_{k_{2}}$ are nonadjacent. By the arbitrariness of $u_{k_{1}}$ and $u_{k_{2}}$, we get that $B_{0}$ is an independent subset of $V(G)$. Similarly, $B_{1}$ is also an independent subset of $V(G)$. It follows that $G$ must be a bipartite graph with two partition sets $B_{0}$ and $B_{1}$. By Claims 3,7, we know that $\left|B_{0}\right|=\left|B_{1}\right|=\delta(G)$. This means that $G \cong K_{\ell, \ell}$, where $\ell=\delta(G)$.

Lemma 2.6. Let $\ell$ be a positive integer. Then $K_{\ell, \ell} \times K_{3}$ is not super- $\kappa$.

Proof. Let $B_{0}=\left\{v_{i} \mid i \in \mathbb{Z}_{\ell}\right\}$ and $B_{1}=\left\{u_{i} \mid i \in \mathbb{Z}_{\ell}\right\}$ be the two partition sets of $K_{\ell, \ell}$. Set $V\left(K_{3}\right)=\mathbb{Z}_{3}$. Let $S=V\left(K_{\ell, \ell}\right) \times\{1\}$. Clearly, $|S|=2 \ell$. By [9], $\kappa\left(K_{\ell, \ell} \times K_{3}\right)=2 \ell$.

Set $W_{0}=\left(B_{0} \times\{0\}\right) \cup\left(B_{1} \times\{2\}\right)$ and $W_{1}=\left(B_{0} \times\{2\}\right) \cup\left(B_{1} \times\{0\}\right)$. Clearly, $V\left(K_{\ell, \ell} \times K_{3}\right)=S \cup W_{0} \cup W_{1}$. It is also easy to see that $\Gamma\left[W_{i}\right] \cong K_{\ell, \ell}$ for $i=0,1$. 
Furthermore, in $K_{\ell, \ell} \otimes K_{3}$ there are no edges between $W_{0}$ and $W_{1}$. It follows that $K_{\ell, \ell} \times$ $K_{3}-S$ is disconnected with no isolated vertices. Therefore, $K_{\ell, \ell} \times K_{3}$ is not super- $\kappa$.

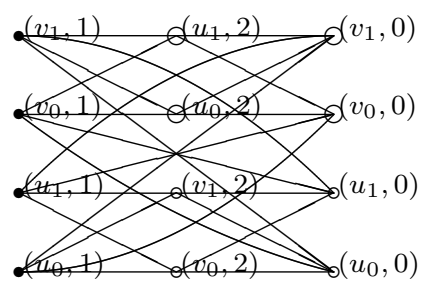

Figure 4: $K_{2,2} \times K_{3}$

Proof of Theorem 1.1. By Lemmas 2.4 and 2.5, we can get the necessity. For the sufficiency, by Lemma $2.6, K_{\ell, \ell} \times K_{3}$ is not super- $\kappa$.

Now assume that $\kappa(\Gamma)=n \kappa(G)$. Suppose to the contrary that $\Gamma$ is super- $\kappa$. Then $\kappa(\Gamma)=\delta(\Gamma)=(n-1) \delta(G)$, and hence $(n-1) \delta(G)=n \kappa(G)$. So, $\kappa(G)<\delta(G)$. Let $T$ be a minimum separating set of $G$. Then $G-T$ has no isolated vertices. By Lemma 2.1 (4), $T \times V\left(K_{n}\right)$ is a separating set of $\Gamma$. Clearly, $\left|T \times V\left(K_{n}\right)\right|=n \kappa(G)$. So, $T \times V\left(K_{n}\right)$ is also a minimum separating set of $G$. Since $\Gamma$ is super- $\kappa, T \times V\left(K_{n}\right)$ must be the neighborhood of some vertex, say $\left(u_{i}, j\right)$. Let $u_{k} \in T$. Then $\left(u_{k}, j\right) \in T \times V\left(K_{n}\right)$, and hence $\left(u_{i}, j\right) \sim\left(u_{k}, j\right)$. This is clearly impossible by the definition of the direct product of graphs. Thus, $\Gamma$ is not super- $\kappa$.

Acknowledgements: The author is indebted to the anonymous referee for many valuable comments and constructive suggestions.

\section{References}

[1] N. Alon and E. Lubetzky, Independent sets in tensor graph powers, J. Graph Theory 54 (2007), $73-87$.

[2] D. Bauer, F. Boesch, C. Suffel and R. Tindell, Connectivity extremal problems and the design of reliable probabilistic networks, in: The Theory and Application of Graphs, Wiley, New York, 1981, 45-54.

[3] F.T. Boesch, Synthesis of reliable networks: A survey, IEEE Trans. Reliability 35 (1986), 240 246.

[4] J.A. Bondy and U.S.R. Murty, Graph Theory with Applications, Elsevier North Holland, New York, 1976.

[5] A. Bottreou and Y. Metivier, Some remarks on the Kronecker product of graphs, Inform. Process. Lett. 68 (1998), 55-61.

[6] B. Brešar, W. Imrich, S. Klavžar and B. Zmazek, Hypercubes as direct products, SIAM J. Discrete Math. 18 (2005), 778-786.

[7] B. Brešar and S. Špacapan, On the connectivity of the direct product of graphs, Austral. J. Combin. 41 (2008), 45-56.

[8] S.A. Ghozati, A finite automata approach to modeling the cross product of interconnection networks, Math. Comput. Model. 30 (1999), 185-200.

[9] R. Guji and E. Vumar, A note on the connectivity of Kronecker product of graphs, Appl. Math. Lett. 22 (2009), 1360-3163. 
[10] L. Guo, C. Qin and X. Guo, Super connectivity of Kronecker product of graphs, Inform. Process. Lett. 110 (2010), 659-661.

[11] R. Hammack, W. Imrich and S. Klavžar, Handbook of Product Graphs, Second Edition, CRC Press 2011.

[12] R.H. Lammprey and B.H. Barnes, Products of graphs and applications, Model. Simul. 5 (1974), 1119-1123.

[13] J. Leskovec, D. Chakrabarti, J. Kleinberg, C. Faloutsos and Z. Gharamani, Kronecker graphs: an approach to modeling networks, J. Mach. Learn. Res. 11 (2010), 985-1042.

[14] A. Mamut and E. Vumar, Vertex vulnerability parameters of Kronecker product of complete graphs, Inform. Process. Lett. 106 (2008), 258-262.

[15] G. Mekiš, Lower bounds for the domination number and the total domination number of direct product graphs, Discrete Math. 310 (2010), 3310-3317.

[16] J. Nešetřil, Representations of graphs by means of products and their complexity, in: Mathematical Foundations of Computer Science, in: Lecture Notes in Comput. Sci. 118, Springer, Berlin, 1981, 94-02.

[17] Y. Wang and B. Wu, Proof of a conjecture on connectivity of Kronecker product of graphs, Discrete Math. 311 (2011), 2563-2565.

[18] W. Wang and N.-N. Xue, Connectivity of direct products of graphs, arXiv: 1102.5180v1 [math.CO] 25 Feb 2011.

[19] H. Wang, E. Shan and W. Wang, On the super connectivity of Kronecker product of graphs, Inform. Process. Lett. 112 (2012), 402-405.

[20] P.M. Weichsel, The Kronecker product of graphs, Proc. Amer. Math. Soc. 8 (1962), 4-52.

[21] A.N. Whitehead and B. Russell, Principia Mathematica, 2, Cambridge University Press. Cambridge, 1912, p.384. 